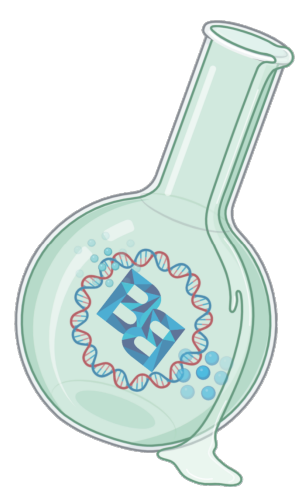

Published by Bio Binge

\title{
Mechanism underlying nuclear encoded plant protein sorting into Mitochondria
}

\author{
Devika $\mathrm{TK}^{1^{*}}$ and Velvili $\mathbf{S}^{1}$ \\ ${ }^{1}$ Department of Biotechnology, Pondicherry University
}

\section{REVIEW}

\begin{abstract}
Mechanism by which the cells increase the mitochondrial mass requires coordinated expression of both the nuclear and organellar genomes and specific intracellular protein targeting, processing and assembly machinery. Mitochondria imports hundreds of different proteins that are encoded by the nuclear genes. Almost $98 \%$ of mitochondrial proteins are synthesized as preproteins in the cytosol. Most of the nuclear encoded mitochondrial proteins are synthesised as precursor proteins containing an $\mathrm{N}$-terminal extension (presequence) which functions as a targeting signal, which is then proteolytically cleaved off after import into mitochondria. These presequences are responsible for the specific targeting of proteins to the matrix of the mitochondria. This review sheds insight into the mechanism underlying the mitochondrial protein import system in plants which encompasses structure and properties of mitochondrial targeting signals, the role of cytoplasmic chaperons in mitochondrial protein targeting, targeting pathway of mitochondrial preproteins and energetics of import process.
\end{abstract}

Keywords: Mitochondrial protein targeting, Presequence, Molecular chaperones, TOM complex, TIM complex, Mitochondrial processing peptidase

\section{Introduction}

Mitochondria is thought to be descended from a single endosymbiotic event approximately 2 billion years ago. Much of the genetic material from the endosymbiont were transferred to the nuclear genome over the course of evolution, thus creating a requirement for the transport and import of these proteins back to their functional site in the organelle $[17,20]$. Mitochondria, known as the powerhouse of the cell, arise by the growth and division of pre-existing mitochondria and this growth occurs by the insertion of newly synthesized constituents, resulting in expansion of the various compartments of each mitochondrion. Mitochondria has an outer membrane, an inner membrane, intermembrane space and an internal compartment (matrix). The main aspect of protein targeting to mitochondria is the transfer of nuclear encoded, cytoplasmically synthesized proteins both across and into the mitochondrial membranes. Hundreds of different mitochondrial proteins participate in these processes [4]. Only a few protein components are encoded by mitochondrial DNA and inserted from the matrix side into the inner membrane. Mitochondrial protein import mechanism in fungi and lower eukaryotes were studied extensively and resulted in characterisation of many signal peptides, protein import machineries on the outer and inner membrane and involvement of various cytosolic and organeller chaperones in the process of transport $[1,2,4]$. The most common type of mitochondrial protein targeting signal is an amino-terminal extension of the preprotein termed as the presequence, which usually consist of 20-50 amino acid residues. These proteins are targeted into the mitochondria with the help of various cytoplasmic molecular chaperons, translocated through the mitochondrial membranes and sorted to different mitochondrial subcompartments. Molecular chaperones mainly helps in translocation, sorting, folding and assembly of newly imported proteins [1,5]. Translocases present in the mitochondrial outer membrane (TOM complex) and in the inner membrane (TIM complex) facilitate recognition of preproteins and transport these preproteins across the two membranes. Once protein has been imported into the mitochondria, they undergo processing 
by mitochondrial processing peptidase (MPP) to form mature proteins [1,4]. The MPP found in plants are membrane-bound metallo-endopeptidase that is integrated into the bc1 complex of the respiratory chain. They consists of two structurally related subunits, $\alpha$ and $\beta$ MPP that cooperate in processing the preprotein. In plants, the MPP was purified from potato, spinach and wheat and was shown to be identical to the core proteins of the bc1 complex. Studies of the mammalian protein indicate that $\beta$-MPP is the catalytic subunit and $\alpha-\mathrm{MPP}$ is necessary for binding the precursor protein and presenting it in the correct conformation. The MPP specifically catalyses the removal of the presequence of a range of different mitochondrial precursor proteins [8]. The mitochondrial protein import is a very complex process including the following events:

(1) Synthesis of the precursor protein in the cytoplasm which contains an $\mathrm{N}$-terminal extension called a presequence which functions as targeting signal.

(2) These precursor protein interacts with various cytosolic chaperones and other factors.

(3) Recognition of the preproteins either through direct interaction of the presequence with organellar import receptors or by the interaction of the presequence bound to cytosolic chaperones.

(4) Translocation of the precursor through the import complexes on organellar membranes.

(5) Proteolytic processing of the precursor inside mitochondria by a highly specific mitochondrial processing peptidase (MPP).

(6) Assembly of the mature form of the protein into a functional, oligomeric protein complex in a process involving organellar chaperones [1,5].

\section{Mitochondrial targeting signals}

Mitochondrial targeting signals are the sequences in preproteins which are necessary to direct the proteins into the mitochondria. Most targeting signals are contained within the $\mathrm{N}$-terminal segments (known as presequences). Since these presequences direct preproteins into the mitochondrial matrix space, presequences are also known as matrix-targeting sequences/signals. Apart from these $\mathrm{N}$-terminal signal, internal targeting sequence are also known to be involved in the translocation of these preproteins into the mitochondria [4]. The size of presequences are highly variable among plants. The size of the cleavable plant mitochondrial presequence ranges from 13 amino acids for the alternative oxidase from Arabidopsis thaliana to 85 amino acids of the $P$ subunit of glycine decarboxylase from pea. Compared with mammalian, yeast and Neurospora crassa presequences, the plant presequences are about 7-9 residues longer [1]. The majority of presequences vary in size from 20 to 60 residues. The plant presequences are very rich in amino acids arginine (12.6\%), alanine (12.0\%), serine (17.1\%), leucine $(10.6 \%)$, but low in cysteine (1.0\%), histidine (1.3\%), tryptophan (1.4\%), tyrosine (1.4\%), glutamic acid (1.4\%) and aspartic acid (1.5\%) [1,5]. The amino acid composition of the plant presequence is similar to that of other mitochondrial presequences as they exhibit high content of basic hydroxylated residues and low content of acidic and aromatic residues. A unique feature exhibited by the plant presequence is that they have higher content of serine (17\%) as compared to that of yeast (7\%), mammals $(3 \%)$ or $\mathrm{N}$. crassa $(10 \%)$ [1,5]. Presequences were reported to have the potential to form an amphiphilic-helix (with few exceptions) which is considered as an essential structural feature for mitochondrial protein import in a wide variety of organisms and this amphiphilicity is the general property of the all mitochondrial targeting sequences $[3,5,16]$. In a helical wheel projection, the positively charged residues tend to localize to one side of the helix and the other side contains uncharged and hydrophobic residues [7]. Domain structure analysis of preprotein showed that it has an import domain and a processing domain. Presequences are known to be involved in two distinct events: mitochondrial receptor recognition (by import domain) and processing activity (by processing domain). It was reported that the arginine residues located at the amino-terminal part of the presequence were important for stabilization of the amphiphilic $\alpha$-helices and for electrostatic interaction with negatively charged regions of the translocating outer and inner membrane translocating apparatus [8].

\section{Role of cytoplasmic chaperons in mitochondrial protein targeting}

Cytosolic chaperones facilitate mitochondrial protein import as well as plays a significant role in stress response mechanisms associated with mitochondrial protein import defects. First, 
chaperones keep precursor protein in an unfolded import competent state. Secondly, chaperones may be linked to mitochondrial receptors that helps to transfer precursors to the TOM complex. Finally, chaperones facilitate mitochondrial protein degradation that has failed to translocate. In the presence of increased cytosolic aggregation of mitochondrial precursor proteins, this latter feature is critical [12,15]. Major molecular chaperons involved in mitochondrial protein targeting include Hsp70 proteins or stress seventy chaperone family, Hsp40 proteins, Hsp90 proteins, Hsp60 proteins and Mitochondria Import Stimulation Factor (MSF). Hsp70 protein is required to keep precursors in a soluble and unfolded state for efficient transport through the TOM and the translocase of the inner membrane complexes [13]. Hsp40s is also known as J-proteins due to the presence of the common J-domain. It acts upstream of Hsp70s and they are the main determinants of Hsp70 specificity by acting as modulators of their function [4,11]. MSF is yet another chaperon that help in import of precursor protein into mitochondria. They are mainly involved in maintaining the preprotein in import competent form. It was reported that MSF helps in unfolding of the wheat germ lysate synthesized aggregated precursor proteins and stimulates their import into the mitochondria in an ATP dependent manner $[9,15]$. These cytoplasmic chaperons interact with both mitochondrial as well as chloroplast preproteins and facilitate in their transport. How these preproteins are specifically targeted to the mitochondria but not to the choroplast? It was reported that transit peptide sequences of chloroplast precursor proteins but not mitochondrial precursors were phosphorylated by a plant specific cytosolic protein kinase and that phosphorylated precursors bind to chloroplasts. Dephosphorylation was required to complete the precursor translocation process across the membranes. This phosphorylation and dephosphorylation event of chloroplast precursor proteins might be one reason for specific sorting process between mitochondria and chloroplasts [1,5].

\section{Targeting pathway of mitochondrial preproteins}

The mitochondrial protein import machinery contains a series of proteins that interact with the presequence of a preprotein in a sequential manner for their import into the mitochondrial matrix. TOM and TIM are the translocases of the outer and inner membranes, respectively [2,7]. Preproteins interact with these translocases in a sequential manner for their import into the matrix (Figure 1). Translocase of the outer mitochondrial membrane (TOM) complex is the entry point for nuclear-encoded proteins. The TOM translocase consists of the primary receptors TOM 70 and TOM 20 and the GIP complex formed from TOM 40, TOM 22, TOM 5, TOM 6 and TOM 7. OM 64 is an outer membrane translocase that is specific to plants [2]. A precursor with internal targeting signals interact with cytosolic chaperones Hsp70 and $\mathrm{Hsp9O}$ in the cytosol. The precursor proteins then binds to its main receptor TOM 70 when the chaperones dock onto this receptor. The precursor is transferred to TOM 5 in an ATP-dependent manner and then passes through the transport channel formed by TOM 40. A backup receptor route via TOM 20 and TOM 22 is also possible for this precursor type. Precursor proteins with presequences on the other hand bind first to TOM 20 and then are subsequently transferred to TOM 22, TOM 5 and the TOM 40 translocation channel. Once the precursor proteins passes through the outer membrane at the TOM complex, these preproteins are separated and guided either towards TIM 23 and TIM 22 translocases of the inner membrane (TIMs). Precursor proteins with a presequence are directed to the TIM 23 complex and those with internal targeting signals are directed to the TIM 22 complex by TIM factors of the intermembrane space [6,14]. The TIM 23 translocase consists of TIM 23, TIM 17 and TIM 50 proteins and peripherally associated TIM 44 which provides a membrane anchor for $\mathrm{mtHsp} 70$ and its cofactor Mge1. Precursor proteins are received from the TOM complex via binding to TIM 50 and the intermembrane space domain of TIM 23 and they are then transported through an aqueous pore formed by the membrane domain of TIM 23 and TIM 17 [7,18]. The membrane potential plays a major role in transport of presequences through the channel. The energy derived from ATP hydrolysis by $\mathrm{mtHsp} 70$ drives protein transport to completion. Mitochondrial hsp70 is very important for import process as mitochondria containing mutant forms of the protein may fail to import precursor proteins. Mitochondrial hsp70 have high affinity for unfolded polypeptide chains and it binds tightly to an imported protein as soon as it emerges in the matrix and then hsp70 releases the protein in an ATP-dependent manner. Two models were proposed to explain how ATP hydrolysis by 
mitochondrial hsp70 drives protein import. In both models, hsp70 proteins are associated with the TIM 23 complex which places them onto the translocating polypeptide chain as it emerges into the matrix. In the thermal ratchet model, the translocating preprotein slides back and forth in the TIM 23 complex by means of thermal motion, then Hsp 70 binds to it and prevent it from backsliding and thereby making the movement directional. In the cross-bridge ratchet mode, the hsp70 proteins that bind to the preprotein and undergo a conformational change driven by ATP hydrolysis which actively pulls a segment of the preprotein chain into the matrix. Then a new molecule of hsp70 then binds to the segment just pulled in and repeat the cycle [14]. Once the preproteins reaches the matrix, presequences are removed by the mitochondrial processing peptidase (MPP),a metalloendopeptidase. The function of mitochondrial processing peptidase (MPP) is to remove the $\mathrm{N}$-terminal targeting signal (the presequence) from nuclear-encoded mitochondrial precursor proteins during or shortly after protein import into mitochondria. MPP is a general peptidase as it can act on several hundred mitochondrial precursor proteins $[1,5]$. MPP is also highly specific as it recognises a distinct cleavage site and specifically cleaves off the presequences. This peptidase have two structurally related subunits, $\alpha$-MPP and $\beta$-MPP which helps in preprotein processing. Both subunits are equally essential for processing. $70 \%$ of the subunit is localised in the membrane and $30 \%$ in the matrix. In plants, the MPP was purified from potato tubers, spinach leaves, wheat and spinach roots. The plant MPP resides in the inner mitochondrial and they are integrated into the cytochrome bc1 complex of the respiratory chain. It is known to occur in dimeric form. The plant MPP is active over a broad $\mathrm{pH}$ and broad temperature, $\mathrm{pH} 6-11$ (optimum at $\mathrm{pH}$ ) and $10-50^{\circ} \mathrm{C}$ (with optimum at $35^{\circ} \mathrm{C}$ ) respectively. These cleaved off presequences are then degraded in the mitochondrial matrix [1].

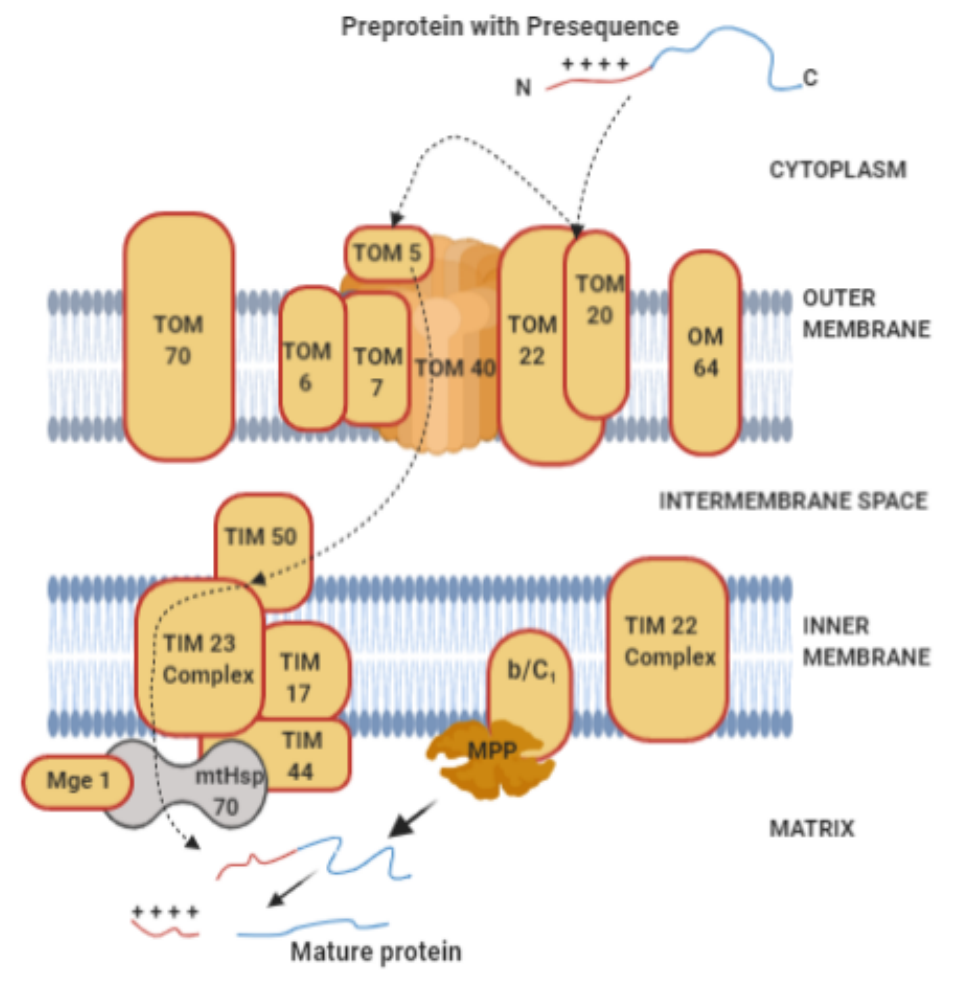

Figure 1. Transport of Preprotein with N-terminal presequence via Translocase of Outer and Inner membrane into the mitochondrial matrix. Precursor protein first binds to TOM 20/TOM 22 and are transferred to TOM5, from where it passes through the pores of major TOM complex, TOM 40 and then via TIM 23, they are imported into the mitochondrial matrix. mtHsp prevent backsliding of precursor in an ATP- dependent manner. The presquence is then cleaved off by the Mitochondrial processing Peptidase (MPP) to form the mature protein.

Light is an important factor that helps in regulating the import of preproteins. Studies carried out using mitochondria isolated from plants at light and darker period of growth reported that 
mitochondria from darker phase imported precursor proteins at much higher ( 4-fold) level than mitochondria isolated from the light period. The inability of mitochondria which were isolated from the light phase to import protein was apparently at the translocation stage of import as binding and processing of the precursor protein was not affected by the time of isolation and this was mainly because certain component of the translocation machinery was either absent or inactivated during the light phase. Another level of control of import occurs at developmental level. In tobacco mitochondria, it was reported that the FAd subunit of the ATP synthase cannot be imported into younger tobacco leaves but can be imported into older tobacco leaves. Developmental regulation of import were also reported with pea leaf mitochondria. Studies on pea leaves showed that a dramatic decrease in import were observed between day 6 and day 30 with pea leaves. This decline in import could be either due to a decline in the level of $\mathrm{mtHsp70}$ as the amount of this protein also decreased over a similar time period or due to other components or factors affecting the activity of $\mathrm{mtH} s p 70$ [1,5].

\section{Carrier Import Pathway}

With reference to protein trafficking, the most notable feature of carrier protein family is the lack of presequence for targeting. The transport of carrier proteins through the cytosol and across the outer membrane is facilitated by molecular chaperones and the TOM complex and their transport to the inner membrane depends on a specialised translocase, the TIM 22 complex. The import of carrier proteins into the inner membrane occurs in 5 stages (Figure 2).

Stage I: The newly synthesised precursor protein binds to molecular chaperones which deliver the precursor to the translocation machinery.

Stage II: The precursor protein binds to the receptor Tom70. Release of preprotein from the receptor is an ATP-dependent process which allows the precursor to transfer to the TOM complex. Stage III: The precursor passes through the translocation channels as hairpin loops making contact with the TIM 9-TIM 10 complex of the intermembrane space.

Stage IV: The precursor inserts into the inner membrane in a membrane potential-dependent manner and this process is assisted by the TIM 22 complex.

Stage V: Finally the precursor proteins assembles into a functional carrier dimer in the inner membrane [14].

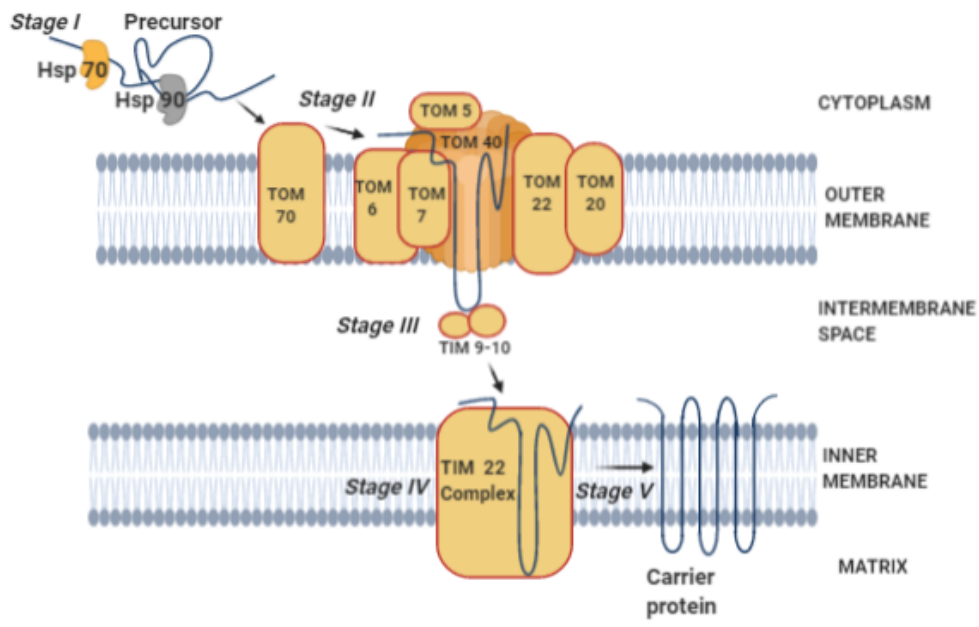

Figure 2. Stages of Import of carrier proteins into the inner membrane. Stage I: precursor protein binds to molecular chaperones. Stage II: the precursor binds to the receptor TOM70, then transferred through TOM complex. Stage III: the precursor passes through the TOM complex as hairpin loops making contact with the TIM9-TIM10 complex. Stage IV: the precursor inserts into the inner membrane, a process assisted by the TIM22 complex. Stage V: precursor assembles into a functional carrier dimer in the inner membrane. 


\section{Energetics of Import process}

Energy from two sources are required for the translocation of precursor proteins across the mitochondrial inner membrane. The first source is the membrane potential $(\delta \psi)$ which plays two roles in precursor import. Membrane potential stimulates the channel protein TIM23 and imparts an electrophoretic effect on presequences. The positively charge presequences are transported through the translocation channel as a result of an electrophoretic effect of $\delta \psi$ (negative on the matrix side). The second source of energy is derived from matrix ATP. Preprotein import into the mitochondrial matrix requires the ATP-dependent action of the molecular chaperone mtHsp70. TIM 44 is an adaptor protein which is located at the TIM23 translocase. It is capable of switching the $\mathrm{mtHsp} 70$ folding function to an important role in protein translocation. The energy obtained from ATP hydrolysis by $\mathrm{mtHsp70}$ drives protein translocation to completion. [14].

\section{Conclusion}

An impressive progress has been made in the past years regarding the mitochondrial protein targeting mechanism in plants. Even though there are a lot of common features in mitochondrial protein targeting mechanism in plants, yeast and mammals, there are several unique features that have been unravelled in plants. Some of these features include characterisation of the plant preprotein presequence, role of various cytoplasmic chaperons involved in efficient targeting of these preproteins into the mitochondria, sorting of these preproteins between mitochondria and chloroplast, role of various outer and inner membrane translocases, role of MPP in preprotein cleavage. Mitochondrial protein target occurs via post translational mechanism. Analysis of presequence length have shown that plant presequence have 7-9 amino acid longer than other species. It has been shown that plant presequences are rich in basic and hydrophobic residues and contain a very few acidic amino acid residues.

The amphiphilic nature of $\mathrm{N}$-terminal region of the preprotein also have been reported which make the presequence to adopt $\alpha$ - helical structure. Plant presequence are rich in basic amino acid Arg, Ala, Leu and less of Cys, His, Trp, Glu, Asp. Serine content is high (17\%) in plant presequence. The $\mathrm{N}$-terminal and $\mathrm{C}$-terminal region of the presequence was found to have more significance in mitochondrial protein targeting than the central region. Domain structure analysis of preprotein showed that it has an import domain and a processing domain. Various cytoplasmic chaperons are found to interact with these preproteins which assist in their folding, prevent aggregation and prevent missorting. Studies have shown that chloroplast preproteins but not mitochondrial preprotein are phosphorylated by plant specific cytoplasmic protein kinases which help in their sorting to chloroplast. Dephosphorylation is also needed for complete preprotein translocation to chloroplast. This phosphorylation and dephosphorylation cycle help in sorting of preprotein between mitochondria and chloroplast. Mitochondrial outer membrane has TOM complex which act as the only entry point for the preproteins. Once it crosses the outer membrane, these preproteins segregate for further import into the matrix through two inner membrane translocases. ATP dependent action of mtHSP70 helps in proper movement of preproteins to mitochondrial matrix. The MPP specifically catalyses the removal of the presequence of a range of different mitochondrial precursor proteins. Membrane potential and ATP hydrolysis play an important role in import of preproteins.

\section{Conflict of interest}

The authors declare that there is no conflict of interest in the subject matter discussed in this manuscript. Both authors contributed equally.

\section{References}

1. Glaser E, Sjöling S, Tanudji M, Whelan J. Mitochondrial protein import in plants. Signals, sorting, targeting, processing and regulation. Plant Mol Biol. 1998; 38(1-2):311-338.

doi:10.1023/a:1006020208140

2. Duncan, O., Murcha, M. W., \& Whelan, J. (2013). Unique components of the plant mitochondrial 
protein import apparatus. Biochimica et biophysica acta, 1833(2), 304-313.

https://doi.org/10.1016/j.bbamcr.2012.02.015

3. Yogev, O., \& Pines, O. (2011). Dual targeting of mitochondrial proteins: mechanism, regulation and function. Biochimica et biophysica acta, 1808(3), 1012-1020.

https://doi.org/10.1016/j.bbamem.2010.07.004

4. Neupert, W. (1997). PROTEIN IMPORT INTO MITOCHONDRIA. Annual Review of Biochemistry, Vol. 66:863-917, 57.

https://doi.org/10.1146/annurev.biochem.66.1.863

5. Glaser E., Whelan J. (2011) Protein Import into Plant Mitochondria. In: Kempken F. (eds) Plant Mitochondria. Advances in Plant Biology, vol 1. Springer, New York, NY.

https://doi.org/10.1007/978-0-387-89781-3_11

6. Fox T. D. (2012). Mitochondrial protein synthesis, import, and assembly. Genetics, 192(4), 1203-1234.

https://doi.org/10.1534/genetics.112.141267

7. Pfanner N. (2000). Protein sorting: recognizing mitochondrial presequences. Current biology: CB, 10(11), R412-R415.

https://doi.org/10.1016/s0960-9822(00)00507-8

8. Sara Sjöling Elzbieta Glaser, Mitochondrial targeting peptides in plants, Trends in plant science, VOLUME 3, ISSUE 4, P136-140, APRIL 01, 1998, https://doi.org/10.1016/S1360-1385(98)01212-6

9. Hachiya, N., Komiya, T., Alam, R., Iwahashi, J., Sakaguchi, M., Omura, T., \& Mihara, K. (1994). MSF, a novel cytoplasmic chaperone which functions in precursor targeting to mitochondria. The EMBO journal, 13(21), 5146-5154

10. Roise, D., Horvath, S. J., Tomich, J. M., Richards, J. H., \& Schatz, G. (1986). A chemically synthesized pre-sequence of an imported mitochondrial protein can form an amphiphilic helix and perturb natural and artificial phospholipid bilayers. The EMBO journal, 5(6), 1327-1334

11. Bykov,Y.S., Rapaport,D.,Herrmann, J.M.,\& S chuldiner,M. (2020). Cytosolic Events in the Biogenesis of Mitochondrial Proteins. Trends in biochemical sciences, 45(8), 650-667.

http://doi.org/10.1016/j.tibs.2020.04.001

12. Kurz, M., Martin, H., Rassow, J., Pfanner, N., \& Ryan, M. T. (1999). Biogenesis of Tim proteins of the mitochondrial carrier import pathway: differential targeting mechanisms and crossing over with the main import pathway. Molecular biology of the cell, 10(7), 2461-2474.

https://doi.org/10.1091/mbc.10.7.2461

13. Komiya, T., Sakaguchi, M., \& Mihara, K. (1996). Cytoplasmic chaperones determine the targeting pathway of precursor proteins to mitochondria. The EMBO journal, 15(2), 399-407

14. Truscott, K. N., Brandner, K., \& Pfanner, N. (2003). Mechanisms of protein import into mitochondria. Current biology: CB, 13(8), R326-R337.

https://doi.org/10.1016/s0960-9822(03)00239-2

15. Boston, R. S., Viitanen, P.V., \& Vierling, E. (1996). Molecular chaperones and protein folding in plants. Plant molecular biology, 32(1-2), 191-222.

https://doi.org/10.1007/BF00039383

16. von Heijne G. (1986). Mitochondrial targeting sequences may form amphiphilic helices. The EMBO journal, 5(6), 1335-1342

17. Murcha, M. W., Kmiec, B., Kubiszewski-Jakubiak, S., Teixeira, P. F., Glaser, E., \& Whelan, J. (2014). Protein import into plant mitochondria: signals, machinery, processing, and regulation. Journal of experimental botany, 65(22), 6301-6335.

https://doi.org/10.1093/jxb/eru399

18. Dekker, P. J., Martin, F., Maarse, A. C., Bömer, U., Müller, H., Guiard, B., Meijer, M., Rassow, J., \& Pfanner, N. (1997). The Tim core complex defines the number of mitochondrial translocation contact sites and can hold arrested preproteins in the absence of matrix Hsp70-Tim 44. The EMBO journal, 16(17), 5408-5419.

https://doi.org/10.1093/emboj/16.17.5408

19. Glover, L. A., \& Lindsay, J. G. (1992). Targeting proteins to mitochondria: a current overview. The Biochemical journal, 284 (Pt 3)(Pt 3), 609-620.

https://doi.org/10.1042/bj2840609 
20. Ghifari, A. S., Huang, S., \& Murcha, M. W. (2019). The peptidases involved in plant mitochondrial protein import. Journal of experimental botany, 70(21), 6005-6018.

https://doi.org/10.1093/jxb/erz365 